\title{
Manganese superoxide dismutase is increased in the airways of smokers' lungs
}

\author{
T. Harju*, R. Kaarteenaho-Wiik*, R. Sirviö*, P. Pääkkö\#, J.D. Crapo*, T.D. Oury ${ }^{+}$, Y. Soini”, \\ V.L. Kinnula
}

Manganese superoxide dismutase is increased in the airways of smokers' lungs. T. Harju, R. Kaarteenaho-Wiik, R. Sirviö, P. Pääkkö, J.D. Crapo, T.D. Oury, Y. Soini, V.L. Kinnula. (C) ERS Journals Ltd 2004.

ABSTRACT: Oxidant stress is a key mechanism for smoking-induced chronic obstructive pulmonary disease (COPD). Smoking has been shown to upregulate several antioxidant enzymes, with potential effects on the prevention of the disease and/or its progression. Superoxide dismutases (SOD)s are the only enzymes capable of consuming superoxide radicals. The purpose of the present study was to investigate SODs in the lungs of nonsmokers, smokers and COPD patients.

Manganese superoxide dismutase (MnSOD), copper zinc SOD (CuZnSOD), and extracellular SOD (ECSOD), were investigated by immunohistochemistry in the airways of 13 nonsmokers, 20 smokers and 22 COPD patients with mild-to-moderate disease. Lung tissue homogenates of three nonsmokers and four smokers were used for Western blot and enzyme activity analysis.

The expression of MnSOD was higher in the central bronchial epithelium of smokers with COPD and in the alveolar epithelium of smokers without or with COPD than in nonsmokers. Lung MnSOD immunoreactivity, evaluated by Western blotting and specific activity, were $33 \%$ and $51 \%$ higher, respectively, in smokers than in nonsmokers. No major changes could be observed in lung CuZnSOD or ECSOD immunoreactivities.

Manganese superoxide dismutase is elevated in the alveolar epithelium of cigarette smokers, probably due to the increased oxidant burden in smokers' lungs.

Eur Respir J 2004; 24: 765-771.

\begin{abstract}
*Dept of Internal Medicine, and ${ }^{\#}$ Dept of Pathology, University of Oulu and Oulu University Hospital, Oulu, and ${ }^{\S}$ Dept of Medicine, Division of Pulmonary Diseases, University of Helsinki and Helsinki University Hospital, Helsinki, Finland. "National Jewish Medical and Research Center, Denver, CO, and ${ }^{+}$Dept of Pathology, University of Pittsburg, Pittsburg, PA, USA.
\end{abstract}

Correspondence: V. Kinnula, Dept of Medicine, BOX 22 (Haartmaninkatu 4), 00014 University of Helsinki, Helsinki, Finland. Fax: 358947176107

E-mail: vuokko.kinnula@helsinki.fi

Keywords: Antioxidant, chronic obstructive pulmonary disease, oxidant, smoking, superoxide dismutase

Received: October 292003

Accepted after revision: June 302004

This study was partly supported by the Finnish Antituberculosis Association Foundation, the Juselius Foundation and the EVO funding of Oulu and Helsinki University Hospitals, Finland.
Smoking is the main aetiological factor causing chronic obstructive pulmonary disease (COPD). A potential mechanism contributing to cigarette smoke-related lung diseases is oxidant stress that overwhelms the primary antioxidant defence of the lung [1-4]. One puff contains $\sim 10^{14}-10^{16}$ of free radicals [4] and the airways of COPD patients contain large amounts of neutrophils, potent generators of free radicals. Cigarette smoke also activates redox-sensitive transcription factors, such as nuclear factor- $\kappa \mathrm{B}$ in COPD [5], which has been shown to regulate the genes of several antioxidant enzymes and related proteins in the lung [6, 7]. A major question in the pathogenesis of COPD is why moderate-tosevere disease develops only in a modest fraction $(\sim 15-20 \%)$ of smokers. One possibility is the variable induction of the primary antioxidant defence in smokers' lungs. The superoxide dismutases (SOD)s are the only superoxide radical scavenging enzymes present in mammalian cells [8, 9]. They are probably key regulators against the oxidant stress induced by cigarette smoke, but their cell-specific expression has never been investigated in the lungs of smokers with or without COPD.

There are three SODs, namely copper zinc SOD (CuZnSOD), manganese SOD (MnSOD) and extracellular SOD (ECSOD). Cytosolic CuZnSOD (SOD1) is thought to function as the primary scavenger of superoxide radicals in the cell cytosol and nucleus. CuZnSOD levels are high in most cells, and studies with CuZnSOD transgenic animals have generally concluded that the protection offered by $\mathrm{CuZnSOD}$ is not significantly improved by $\mathrm{CuZnSOD}$ overexpression [10] and, under most pathophysiological conditions, the natural intracellular levels of CuZnSOD are sufficient. MnSOD (SOD2) is a mitochondrial enzyme known to play an essential role in the oxidant resistance of several vital organs [10]. Overexpression of MnSOD protects alveolar epithelium against oxidative stress, whereas a deficiency of MnSOD is lethal, with MnSOD knockout mice dying within 1-2 weeks after birth [11]. ECSOD (SOD3) is a secretory $\mathrm{Cu}-$ and $\mathrm{Zn}$-containing tetrameric protein of two dimers. In mice, the loss of ECSOD can be sufficiently balanced by other antioxidants and ECSOD knockout mice appear to develop normally, unless the animals are highly stressed. In these knockout animals, ECSOD has been shown to be strongly involved in protecting the lung in inflammatory states [12]. SODs are hypothesised to play an important role in the primary defence of the human lung against free radicals, such as cigarette smoke, and possibly in protecting against the progression of smoking related lung diseases.

In the present study, the distribution and expression of all forms of SODs were investigated by immunohistochemistry at various levels of the airways from nonsmokers, smokers 
without obstruction and smokers with mild-to-moderate COPD. Immunoreactivities of all SODs by Western blotting and lung SOD activities were further analysed from selected samples from nonsmokers, smokers and COPD. Increased oxidant stress, both in the lungs of smokers without the disease and COPD, was also confirmed by one of the fingerprints of oxidative stress, 4-hydroxy-2-noneal (4-HNE), a marker of lipid peroxidation [13].

\section{Material and methods}

Lung tissue samples from 55 patients (22 smokers with COPD, 20 smokers with normal lung function and 13 lifelong nonsmokers) undergoing resection for a lung tumour were drawn from the archives of the Dept of Pathology, Oulu University Hospital, Oulu, Finland. Given that the resection of malignant tumours may, theoretically, have an influence on the adjacent structures, lung tissue samples of nonmalignant lung obtained during surgery for hamartomas were additionally included (one in the nonsmoker group and two in the COPD group). Tissue specimens from tumour-free central bronchi and peripheral lung tissue were selected. The smokers had either normal lung function or they had mild-tomoderate COPD with a bronchodilatation response $<12 \%$. The patients were not receiving corticosteroid therapy and did not suffer from asbestos-related disease. The clinical characteristics were obtained from the patient records (table 1). All 55 cases were included in the MnSOD and CuZnSOD immunohistochemistry, 39 in the ECSOD and 30 in the 4HNE immunohistochemistry.

Additional seven lung tissue samples from separate individuals (stored in liquid nitrogen) were used for Western blot and enzyme activity analysis. They included three lifelong nonsmokers (mean age $68 \mathrm{yrs}$ ) and four smokers with a Global Initiative for Chronic Obstructive Lung Disease (GOLD) Stage 0-II COPD (mean age 66 yrs and 50-78 pack-yr history of smoking).

\section{Immunohistochemistry}

Two tissue blocks from each patient were selected, one from the resection line with a central cartilage-containing bronchus and the other from the peripheral lung. For immunohistochemical analysis, $4-\mu \mathrm{m}$ sections were cut. Immunohistochemistry was conducted with aminoethyl carbazole (Zymed Laboratories Inc., San Francisco, CA, USA) as described by LAKARI et al. [14]. The primary polyclonal antibodies for CuZnSOD, MnSOD, ECSOD [15-17] and 4HNE (Calbiochem Co., La Jolla, CA, USA) with dilutions of 1:200 for CuZnSOD, 1:1,000 for MnSOD, 1:200 for ECSOD and 1:250 for 4-HNE were used. The immunostaining was done using the Histostain-Plus Kit (Zymed Laboratories Inc.). In negative controls, the primary antibodies were substituted with PBS or rabbit primary antibody isotype control (Zymed Laboratories Inc.).

Immunoreactivity was assessed semiquantitatively by grading the staining intensity of epithelium or macrophages as follows: 0) negative; 1) weak; 2) moderate; or 3) intense. All analyses were conducted independently by two experienced lung pathologists, and interobserver repeatability, measured using Cohen's kappa statistics, was moderate $(\kappa=0.496$, $\mathrm{p}=0.0001$ ).

\section{Western blotting}

Lung tissue samples $(n=7)$ frozen in liquid nitrogen were homogenised in PBS ( $\mathrm{pH}$ 7.4). A total of $50 \mu \mathrm{g}$ of cell protein per lane was applied to a $12 \%$ SDS-PAGE and the gel was electrophoresed as previously described [18]. The blotted membrane was incubated with the antibody to CuZnSOD (diluted 1:10,000), MnSOD (diluted 1:10,000) or to ECSOD (diluted 1:2,500) followed by an anti-rabbit antibody conjugated to horseradish peroxidase (dilution 1:30,000; Amersham, Freiburg, Germany). CuZnSOD, MnSOD and ECSOD expressions of the patients and normal control subjects were compared quantitatively by scanning densitometry using 300A Computing Densitometer and Image Quant Software v3.0 Fast Scan (Molecular Dynamics, Sunnyvale, CA, USA). Given the evident problems with $\beta$-actin and glyceraldehyde- 3 '-phosphate dehydrogenase in obstructive lung diseases [19], they were not used as housekeeping genes/loading controls in the present study. Instead, the loading homogeneity was confirmed by careful protein measurement of the samples.

\section{Superoxide dismutase activity}

Tissue samples preserved in liquid nitrogen were homogenised in PBS. Total SOD activity was immediately measured by spectrophotometry by the method of MCCORD and FRIDOVICH [8]. MnSOD was distinguished from CuZnSOD by its resistance to $1 \mathrm{mM}$ potassium cyanide. The activity is expressed as units $\cdot \mathrm{mg}^{-1}$ protein.

Table 1.-Patient characteristics subdivided according to smoking habit and the presence of COPD in the immunohistochemistry part of the study

$$
\text { Nonsmokers } \quad \text { Smokers without COPD }
$$

\begin{tabular}{|c|c|c|c|c|}
\hline Subjects $n$ & 13 & 20 & 22 & \\
\hline Age yrs & $66 \pm 12$ & $62 \pm 8$ & $65 \pm 7$ & 0.411 \\
\hline Sex male:female & $5: 8$ & $16: 4^{\#}$ & $21: 1^{\bullet}$ & $0.001^{+}$ \\
\hline Pack-yrs & 0 & $48 \pm 19^{\#}$ & $43 \pm 12$ & 0.002 \\
\hline Height $\mathrm{m}$ & $1.65 \pm 0.09$ & $1.72 \pm 0.09$ & $1.72 \pm 0.06$ & 0.034 \\
\hline Weight kg & $71 \pm 15$ & $82 \pm 20$ & $76 \pm 18$ & 0.215 \\
\hline FEV1 L & $2.75 \pm 0.85$ & $3.11 \pm 0.68^{\S}$ & $2.18 \pm 0.36^{\circ}$ & 0.000 \\
\hline Range & $1.74-4.10$ & $1.65-4.24$ & $1.53-2.95$ & \\
\hline FEV1 \% pred & $97.0 \pm 12.5$ & $91.2 \pm 8.7^{\S}$ & $63.7 \pm 8.0$ & 0.000 \\
\hline FEV $1 / \mathrm{FVC} \%$ & $88.0 \pm 10.1$ & $82.9 \pm 11.0^{\S}$ & $63.9 \pm 8.9^{\bullet}$ & 0.000 \\
\hline
\end{tabular}

\begin{tabular}{|c|c|c|c|c|}
\hline Subjects $n$ & 13 & 20 & 22 & \\
\hline Age yrs & $66 \pm 12$ & $62 \pm 8$ & $65 \pm 7$ & 0.411 \\
\hline Sex male:female & $5: 8$ & $16: 4^{\#}$ & $21: 1^{\bullet}$ & $0.001^{+}$ \\
\hline Pack-yrs & 0 & $48 \pm 19^{\#}$ & $43 \pm 12$ & 0.002 \\
\hline Height $\mathrm{m}$ & $1.65 \pm 0.09$ & $1.72 \pm 0.09$ & $1.72 \pm 0.06$ & 0.034 \\
\hline Weight kg & $71 \pm 15$ & $82 \pm 20$ & $76 \pm 18$ & 0.215 \\
\hline FEV1 L & $2.75 \pm 0.85$ & $3.11 \pm 0.68^{\S}$ & $2.18 \pm 0.36^{\circ}$ & 0.000 \\
\hline Range & $1.74-4.10$ & $1.65-4.24$ & $1.53-2.95$ & \\
\hline FEV1 \% pred & $97.0 \pm 12.5$ & $91.2 \pm 8.7^{\S}$ & $63.7 \pm 8.0$ & 0.000 \\
\hline FEV $1 / \mathrm{FVC} \%$ & $88.0 \pm 10.1$ & $82.9 \pm 11.0^{\S}$ & $63.9 \pm 8.9^{\bullet}$ & 0.000 \\
\hline
\end{tabular}

Smokers with COPD

ANOVA p-value

Data are presented as mean \pm SD. COPD: chronic obstructive pulmonary disease; FEV1: forced expiratory volume in one second; FVC: forced vital capacity. ${ }^{\#}$ : comparison between nonsmokers and smokers without COPD significant at the $\mathrm{p}=0.05$ level; ${ }^{\text {": }}$ comparison between nonsmokers and smokers with COPD significant at the $\mathrm{p}=0.05$ level; ${ }^{\S}$ : comparison between smokers without COPD and with COPD significant at the $\mathrm{p}=0.05$ level; ${ }^{+}$: Fisher's exact test. 


\section{Statistical analyses}

Continuous data were compared using ANOVA. When ANOVA results indicated that groups differed, post hoc comparisons were performed using two-tailed paired t-tests. Categorical data were compared using Fisher's exact test designed for small sample groups. A p-value $<0.05$ was considered statistically significant.

\section{Ethical considerations}

The study protocol was approved by the ethical committee of the University of Oulu and Oulu University Hospital, Oulu, Finland.

\section{Results}

\section{Manganese superoxide dismutase}

When analysed from the whole material, the expression of MnSOD was most intense in the central bronchial epithelium and in the alveolar macrophages, the difference being statistically significant between central bronchial epithelium and alveolar epithelium ( $\mathrm{p}=0.034)$, as well as between alveolar macrophages and alveolar epithelium $(\mathrm{p}=0.035$; analysed by Fisher's exact test).

MnSOD intensity in the large airways of nonsmokers varied from negative or weak (10 out of 13 ) to moderate (three out of 13), and in small bronchioli from weak (nine out of 13) to moderate or intense (four out of 13). Vascular endothelial cells and alveolar epithelium showed no immunoreactivity. Alveolar macrophages were weakly positive in seven out of 13 and moderate or intense in six out of 13 cases. On average, $17 \%(\mathrm{SD}: 23 \%)$ of macrophages were positive (fig. 1a-d, and fig. 2, $\square$ ).

When the nonsmokers and smokers without COPD (average 48 pack-yrs) were compared, MnSOD immunoreactivity was clearly elevated in the alveolar epithelium of smokers' lungs $(p=0.022)$. MnSOD did not differ in the central bronchial and peripheral bronchiolar epithelium, alveolar macrophages or blood vessels. MnSOD expression was significantly higher in the central bronchial epithelium $(\mathrm{p}=0.041)$, especially in the preserved alveolar epithelium $(\mathrm{p}=0.010)$ in COPD patients compared to the nonsmokers, but again there was no corresponding difference in peripheral bronchiolar epithelium or in alveolar macrophages. When the immunoreactivities were compared between the smokers and subjects with COPD, MnSOD immunoreactivity in the central bronchial epithelium, alveolar epithelium, alveolar macrophages or blood vessels did not differ significantly. A summary of the expression of MnSOD at various airway levels of nonsmokers, smokers and COPD patients is presented in figure 2.

Assessment of the enzyme from the total lung homogenate does not reflect the enzyme content in the critical cell types of the lung. However, there was a tendency for higher MnSOD immunoreactivity observed by Western blotting analysis in smokers (four patients) compared to nonsmokers (three cases), this difference being $33 \%$ by densitometry (fig. 3). Furthermore, the specific activity of MnSOD was 51\% higher in smokers when compared to the corresponding value in nonsmokers.

\section{Copper zinc superoxide dismutase}

$\mathrm{CuZnSOD}$ immunohistochemistry revealed weak immunoreactivity in the central bronchial epithelium in two out of 13 nonsmokers, 10 out of 20 smokers and 11 out of 22 COPD patients. Other cases were negative with the exception of one COPD, in whom there was moderate immunoreactivity. The peripheral bronchiolar epithelium showed weak immunoreactivity in five out of 13 nonsmokers, four out of 20 smokers and four out of 22 COPD patients. Others were negative with the exception of one nonsmoker who exhibited moderate immunoreactivity. Alveolar epithelium was mainly negative. The alveolar macrophages showed weak immunoreactivity in some macrophages (usually $<5 \%$; fig. 1e). The differences in the immunoreactivities between the patient groups were not significant. Nondetectable CuZnSOD reactivity in some cases can be related to the low sensitivity of immunohistochemistry to detect minor amounts of enzyme proteins. However, CuZnSOD was also assessed by Western blotting and activity analysis. Western blotting of CuZnSOD suggested higher CuZnSOD immunoreactivity in smokers compared to nonsmokers $(+25 \%$, mean from the densitometry analysis of four patients, not significant; fig. 3). There was also no significant change in the lung $\mathrm{CuZnSOD}$ activity in these same patients (31.3 16.6 units $\cdot \mathrm{mg}^{-1}$ protein) compared to nonsmokers $\left(26.2 \pm 6.8\right.$ units $\cdot \mathrm{mg}^{-1}$ protein $)$.

\section{Extracellular superoxide dismutase}

By immunohistochemistry, ECSOD immunoreactivity was negative in the peripheral bronchioli in 11 out of 13 nonsmokers, eight out of 11 smokers and 11 out of 15 COPD patients. Other cases showed weak immunoreactivity for ECSOD. Alveolar macrophages were mainly ECSOD negative or weakly stained. Only the positive blood vessel walls per field differed significantly between the groups: nonsmokers showed more often positive immunoreactivity $(1.15 \pm 0.33)$ than smokers with normal lung function $(0.44 \pm 0.12)$ or COPD patients $(0.28 \pm 0.07, \mathrm{p}=0.011$, fig. 1f). The antibody used is highly specific for ECSOD [17], which was also confirmed by the Western analysis. Western blotting showed no major changes in ECSOD immunoreactivity in the nonsmokers and smokers $(21 \%$ increase compared to controls; fig. 3). The specific activity of ECSOD could not be measured due to exhaustion of the samples.

\section{4-hydroxy-2-noneal}

COPD has already been shown to lead to elevated levels of 4-HNE expression as a marker of lipid peroxidation [13]. The present study confirmed higher 4-HNE levels in alveolar macrophages, both in smokers without and with COPD, compared to nonsmokers ( $\mathrm{p}=0.027)$, the percentage of positive macrophages also being higher $(\mathrm{p}=0.004)$. 4-HNE staining in the preserved alveolar epithelium in the various groups of patients did not differ significantly.

\section{Discussion}

Previous studies, as well as the present study, show that the oxidant burden of smoker's lung and/or those with COPD is significantly increased [2, 3, 13]. The reason why COPD develops in a small fraction of smokers may be related to the cell and site-specific regulation of the major antioxidant defence mechanisms in the lung, and not only because of the increased oxidant stress in general. A recent microarray study showed no significant induction of SODs in the large airways of smokers with the ratio of the gene expression in smokers/ nonsmokers varying between $0.80-1.26$ [20]. However, a 

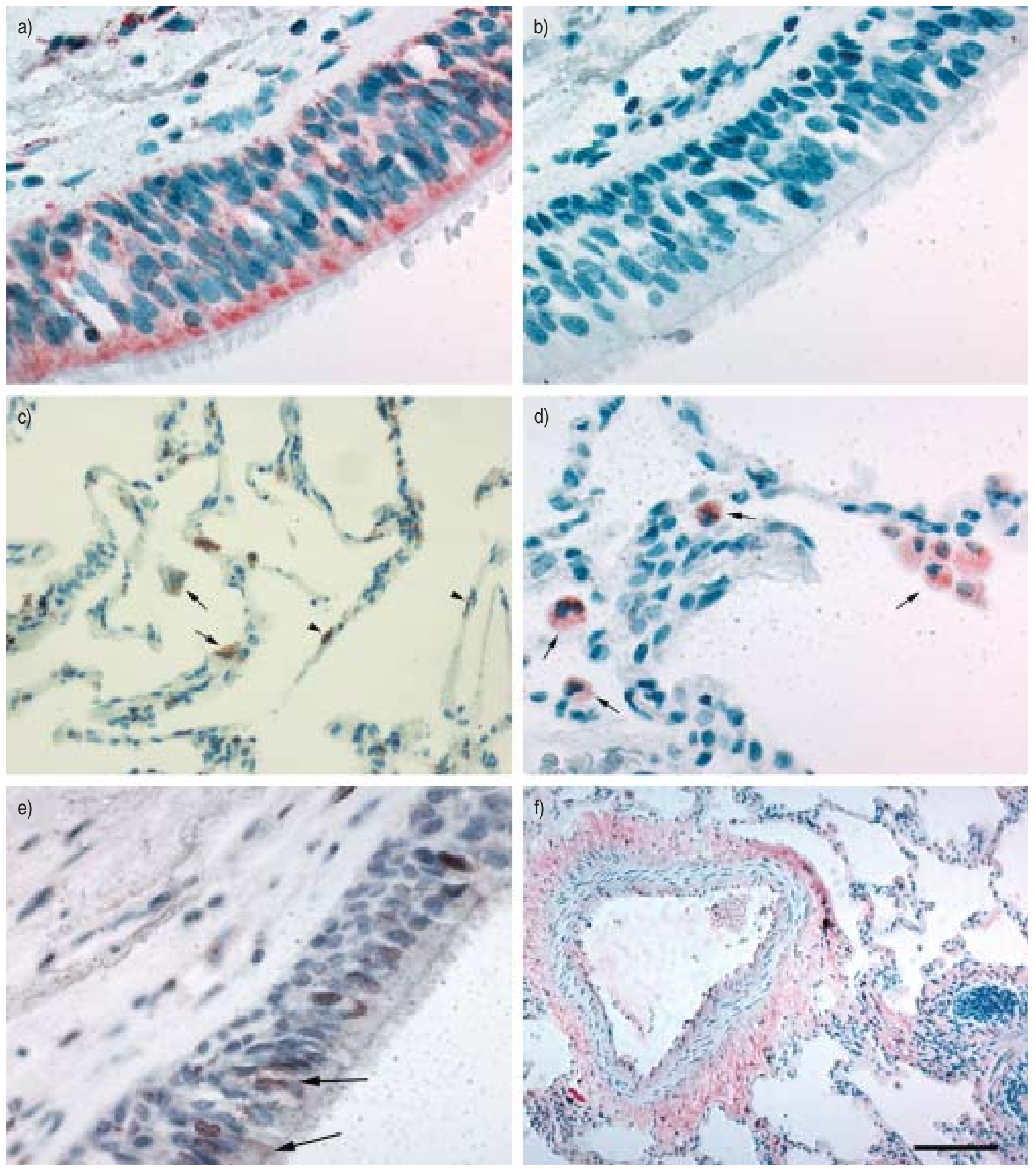

Fig. 1.-a) Moderate expression of manganese (Mn) superoxide dismutase (SOD) in the epithelium of central bronchus in chronic obstructive pulmonary disease (COPD). b) Rabbit serum control without the antibody. c) Weak expression of MnSOD in alveolar epithelium of a COPD patient in cuboidal alveolar type II pneumocytes (arrows) and also in cells with typical localisation for type I pneumocytes (arrowheads). d) Moderate expression of MnSOD in alveolar macrophages (arrows) from a COPD patient. e) Weak expression of copper zinc SOD in the epithelial cells (arrows) of central bronchus in COPD. f) Positive immunostaining showing extracellular (EC) SOD immunoreactivity in peripheral pulmonary vessel (artery) in a COPD patient. In contrast to the other cases, in this particular case, the alveolar epithelium showed moderate immunoreactivity for ECSOD. Scale bar $=40 \mu \mathrm{m}$.

remarkable individual variability could be observed, possibly suggesting variation in the susceptibility to the development of COPD. The present study assessed the protein levels of all SODs in the critical areas of the lung, which provides an accurate depiction of cigarette smoke-induced changes of these enzymes in the human lung. The current study clearly demonstrates that the localisation of these enzymes in the lung is variable. In general, they appear to be concentrated 


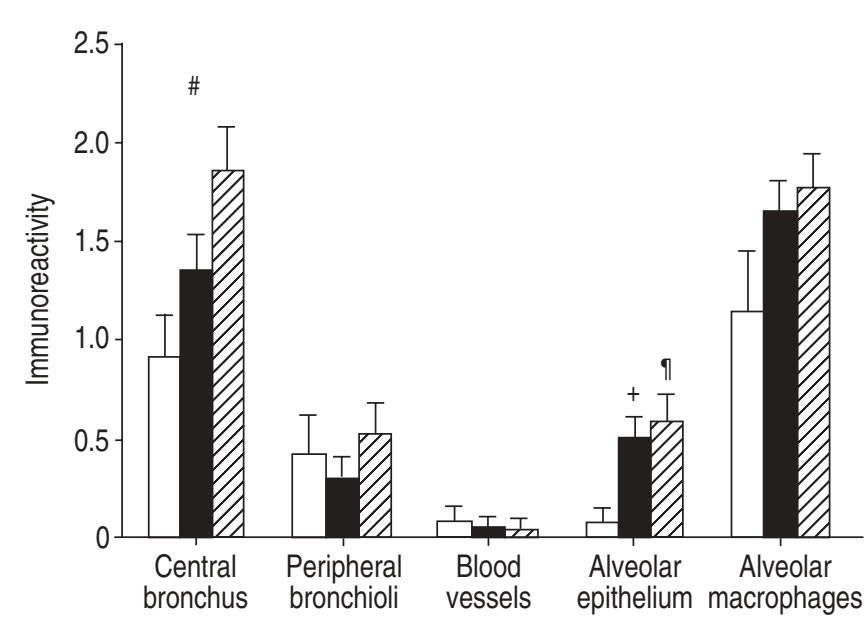

Fig. 2.-Expression of manganese superoxide dismutase (MnSOD) in the epithelium of central bronchus, peripheral bronchiolus, blood vessels, alveolar epithelial cells and alveolar macrophages in nonsmokers $(\square)$, smokers with normal lung function ( $\square$ ) and smokers with chronic obstructive pulmonary disease (COPD; $\mathbb{Z}$ ). Immunoreactivity was assessed from the biopsies of 13 nonsmokers, 20 smokers and 22 smokers with COPD. Data are presented as mean \pm SEM. ${ }^{\#}$ : $\mathrm{p}=0.041$, nonsmokers versus smokers with COPD; ${ }^{\uparrow}: \mathrm{p}=0.01$, nonsmokers versus smokers with COPD; ${ }^{+}: \mathrm{p}=0.022$, nonsmokers versus smokers with normal lung function.

$\mathrm{kD}$
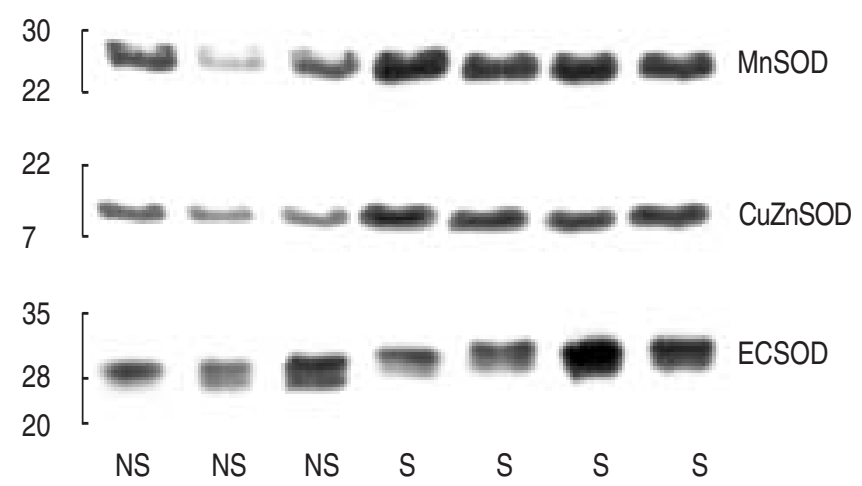

Fig. 3.-Western blotting analyses showing manganese (Mn) superoxide dismutase (SOD), copper $(\mathrm{Cu})$ zinc $(\mathrm{Zn})$ SOD, and extracellular (EC) SOD immunoreactivities in lung tissue homogenates of nonsmokers (NS) and smokers (S). Immunoreactivity of CuZnSOD is $25 \%$ higher in smokers (mean from four patients, densitometry) than in nonsmokers. Mean of MnSOD immunoreactivity is 33\% higher in smokers than in nonsmokers. Mean of ECSOD immunoreactivity shows a slight tendency for higher immunoreactivity $(21 \%)$ in smokers than in nonsmokers.

more in the large airways and alveolar macrophages than in small airways. The presented results suggest that MnSOD is induced in smoker's lung, not only in the central bronchial epithelium but even more importantly in the alveolar epithelium.

Cell-specific expression of MnSOD has been detected in the human lung [14], but its immunoreactivity has not been previously compared at various levels of the airways. Consistent with the previous findings, MnSOD was expressed in central bronchial epithelium, as well as in alveolar macrophages, but the expression was much weaker in peripheral airways than in the bronchial epithelium of large airways. This difference may have important effects on the development of lung diseases with peripheral tissue damage, such as emphysema.

MnSOD mRNA, protein and/or activity are induced in various cells, including lung cells, by oxidants, cytokines, asbestos fibres, modulators of the cellular redox state and cigarette smoke [9]. Only a few studies have investigated MnSOD in human lung diseases. MnSOD is elevated in the inflammatory phases of interstitial lung diseases [14, 16]. In contrast, the RNA levels of MnSOD appear to be very similar in the central airways of nonsmokers and smokers [20]. However, in the present study, immunoreactivity of MnSOD, especially in the alveolar epithelium of smokers and patients with mild-to-moderate COPD, was higher than in nonsmokers, and this same tendency could also be observed with Western blotting and activity analysis. This suggests that MnSOD can be induced during prolonged oxidant exposures, especially in the alveolar epithelium of smoker's lung. The current study raises the possibility that SOD (by induction in some individuals or by exogenous delivery) could perhaps protect against progressive and severe COPD.

CuZnSOD is the major cytosolic SOD, being mainly expressed in bronchial epithelium of the human lung [16, 21]. There are studies suggesting that CuZnSOD mRNA can be upregulated by oxidants, metal ions and radiation in vitro [22]. However, most studies have not detected any changes in lung $\mathrm{CuZnSOD} \mathrm{mRNA}$ or activity in response to cytokines or oxidant stress in experimental animal models in vivo, cell studies in vitro [23-26], or in cigarette smokers [20]. The level of CuZnSOD also appears to be very constant during lung development [27], in parenchymal lung diseases [9, 14] and lung malignancies [28], but it has been suggested to decrease in asthmatic airways [29]. Consistent with most previous studies, CuZnSOD immunohistochemistry showed no marked differences between smokers and nonsmokers in the current study, neither was the activity of $\mathrm{CuZnSOD}$ significantly changed in smoker's lung. Some specimens were negative for $\mathrm{CuZnSOD}$ by immunohistochemistry, but this does not exclude the existence of the enzyme in those areas. Overall, the results, with three various methodologies i.e. immunohistochemistry, Western blotting and activity, were consistent and suggest no major alterations of CuZnSOD in smoker's lung or mild-to-moderate COPD.

ECSOD is the main SOD in pulmonary and systemic vessels [17]. ECSOD is expressed in the lung, especially in the extracellular matrix and vessels, and to a lesser degree in the bronchial epithelium, alveolar epithelium and alveolar macrophages $[17,30]$. ECSOD has been suggested to be of major importance, especially against extracellular free radicals. ECSOD is known to be induced to some extent by inflammatory cytokines, but ECSOD may also be proteolytically cleaved in various pathological conditions in vivo [30]. However, immunohistochemical assessment of ECSOD is difficult since the enzyme is expressed widely in the extracellular space where in situ hybridisation and immunoelectron microscopy have been given more precise locations $[9,30]$. In the present study, ECSOD was also confirmed by Western blotting analysis. In agreement with a recent microarray study on smokers [20], no major changes of lung ECSOD immunoreactivity could be found in smoker's lung. One interesting finding was the higher immunoreactivity of ECSOD in the vessels of nonsmokers compared with smokers. This raises interesting questions about the role of ECSOD in smokinginduced atherosclerosis.

It can be hypothesised that the low response to exogenous oxidants and/or lack of SOD inducibility and/or genetic variability, mainly their polymorphisms, may predispose an individual to the development of COPD. Experimental studies on rats have shown lung MnSOD induction after acute exposure to cigarette smoke [31], and that after initial 
induction, MnSOD may also be downregulated by severe oxidant stress and during apoptosis [23]. Thus, long-lasting smoking could also downregulate SODs in the human lung. The present study does not exclude the possibility of SOD downregulation in severe COPD. Since the present study included smokers and patients with mild-to-moderate disease, further studies will be needed to compare the levels of these enzymes in smokers at various stages of the disease. There is also need for further studies on the individual variability of MnSOD in smoker's lung.

The best documented host factor that predisposes to the development of COPD is hereditary $\alpha$-1-antitrypsin deficiency. Other genes involved in the pathogenesis of COPD may include detoxification enzymes and other related mechanisms, but their clinical significance is currently unclear. Exposure to tobacco smoke and to a lesser extent to other outdoor or indoor pollutants, occupational dust and chemicals, infections, low socioeconomic status, as well as poor dietary habits, have been thought to influence the risk for COPD. The interaction between host factors and environmental exposures together contribute to the development of COPD.

The pathogenesis of COPD is clearly associated with increased oxidant stress, and rather the oxidant/antioxidant balance in general than one individual enzyme. One marker of oxidant related injury is 4-HNE, which has been shown to be elevated both in the alveolar macrophages and alveolar epithelium of COPD [13]. The presented results with 4-HNE and alveolar macrophages are consistent with that recent study by RAHMAN et al. [13]. However, the current authors could not show significant differences in the 4-HNE staining in the preserved alveolar epithelium of nonsmokers, smokers without COPD and smokers with mild-to-moderate COPD. This finding may be related to several factors, suggesting that various antibodies, staining methods and diseases of different severity can give variable results. Highly sensitive markers of oxidant-mediated cell and tissue injury will still be needed.

In addition to MnSOD, the levels of hydrogen peroxide $\left(\mathrm{H}_{2} \mathrm{O}_{2}\right)$-scavenging enzymes can also be modulated in COPD. The lack of inducibility and/or downregulation of these enzymes may further enhance the progression of cigarette smoke-related lung injury. There are studies showing that several genes of the glutathione pathway are upregulated in smoker's lung [20,32], although in these studies the protein expressions or functional enzyme activities were not analysed. In contrast, the current authors [1] and others [33] have shown that the immunoreactive protein of $\gamma$-glutamyl cysteine synthetase was lower in smoker's lung and COPD than in nonsmokers. To the knowledge of the present authors, no other $\mathrm{H}_{2} \mathrm{O}_{2}$-scavenging antioxidant enzymes have been investigated in COPD with variable disease severity.

Overall, it seems that there is a complex balance of oxidants and antioxidants playing a crucial role in the pathogenesis of smoking-related lung diseases.

Acknowledgements. The authors are grateful to M. Tuovinen for his excellent technical assistance.

\section{References}

1. Harju T, Kaarteenaho-Wiik R, Soini Y, Sormunen R, Kinnula VL. Diminished immunoreactivity of gammaglutamylcysteine synthetase in the airways of smokers' lung. Am J Respir Crit Care Med 2002; 166: 754-759.

2. Ichinose M, Sugiura H, Yamagata S, Koarai A, Shirato K. Increase in reactive nitrogen species production in chronic obstructive pulmonary disease airways. Am J Respir Crit Care Med 2000; 162: 701-706.

3. Rahman I, MacNee W. Role of oxidants/antioxidants in smoking-induced lung diseases. Free Radic Biol Med 1996; 21: 669-681.

4. Nakayama T, Church DF, Pryor WA. Quantitative analysis of the hydrogen peroxide formed in aqueous cigarette tar extracts. Free Radic Biol Med 1989; 7: 9-15.

5. Di Stefano A, Caramori G, Oates $\mathrm{T}$, et al. Increased expression of nuclear factor-kappaB in bronchial biopsies from smokers and patients with COPD. Eur Respir J 2002; 20: $556-563$.

6. Brady TC, Chang LY, Day BJ, Crapo JD. Extracellular superoxide dismutase is upregulated with inducible nitric oxide synthase after NF-kappa B activation. Am J Physiol 1997; 273: Suppl. 5, L1002-L1006.

7. Comhair SA, Erzurum SC. Antioxidant responses to oxidant-mediated lung diseases. Am J Physiol Lung Cell Mol Physiol 2002; 283: L246-L255.

8. McCord JM, Fridovich I. Superoxide dismutase. An enzymic function for erythrocuprein (hemocuprein). J Biol Chem 1969; 244: 6049-6055.

9. Kinnula VL, Crapo JD. Superoxide dismutases in the lung and human lung diseases. Am J Respir Crit Care Med 2003; 167: $1600-1619$.

10. Tsan MF. Superoxide dismutase and pulmonary oxygen toxicity: lessons from transgenic and knockout mice. Int J Mol Med 2001; 7: 13-19.

11. Ilizarov AM, Koo HC, Kazzaz JA, et al. Overexpression of manganese superoxide dismutase protects lung epithelial cells against oxidant injury. Am J Respir Cell Mol Biol 2001; 24: 436-441.

12. Carlsson LM, Jonsson J, Edlund T, Marklund SL. Mice lacking extracellular superoxide dismutase are more sensitive to hyperoxia. Proc Natl Acad Sci USA 1995; 92: 6264-6268.

13. Rahman I, van Schadewijk AA, Crowther AJ, et al. 4Hydroxy-2-nonenal, a specific lipid peroxidation product, is elevated in lungs of patients with chronic obstructive pulmonary disease. Am J Respir Crit Care Med 2002; 166: 490-495.

14. Lakari E, Pääkkö P, Pietarinen-Runtti P, Kinnula VL Manganese superoxide dismutase and catalase are coordinately expressed in the alveolar region in chronic interstitial pneumonias and granulomatous diseases of the lung. $\mathrm{Am}$ J Respir Crit Care Med 2000; 161: 615-621.

15. Crapo JD, Oury T, Rabouille C, Slot JW, Chang LY. Copper, zinc superoxide dismutase is primarily a cytosolic protein in human cells. Proc Natl Acad Sci USA 1992; 89: 10405-10409.

16. Lakari E, Pääkkö P, Kinnula VL. Manganese superoxide dismutase, but not $\mathrm{CuZn}$ superoxide dismutase, is highly expressed in the granulomas of pulmonary sarcoidosis and extrinsic allergic alveolitis. Am J Respir Crit Care Med 1998; 158: 589-596.

17. Oury TD, Chang LY, Marklund SL, Day BJ, Crapo JD. Immunocytochemical localization of extracellular superoxide dismutase in human lung. Lab Invest 1994; 70: 889-898.

18. Jarvinen $\mathrm{K}$, Pietarinen-Runtti $\mathrm{P}$, Linnainmaa $\mathrm{K}$, et al. Antioxidant defense mechanisms of human mesothelioma and lung adenocarcinoma cells. Am J Physiol 2000; 278: L696-L702.

19. Glare EM, Divjak M, Bailey MJ, Walters EH. beta-Actin and GAPDH housekeeping gene expression in asthmatic airways is variable and not suitable for normalising mRNA levels. Thorax 2002; 57: 765-770.

20. Hackett NR, Heguy A, Harvey BG, et al. Variability of antioxidant-related gene expression in the airway epithelium of cigarette smokers. Am J Respir Cell Mol Biol 2003; 29: 331-343.

21. Kinnula VL, Yankaskas JR, Chang L, et al. Primary and immortalized (BEAS 2B) human bronchial epithelial cells have significant antioxidative capacity in vitro. Am J Respir Cell Mol Biol 1994; 11: 568-576. 
22. Zelko IN, Mariani TJ, Folz RJ. Superoxide dismutase multigene family: a comparison of the CuZn-SOD (SOD1), Mn-SOD (SOD2), and EC-SOD (SOD3) gene structures, evolution, and expression. Free Radic Biol Med 2002; 33: 337-349.

23. Clerch LB, Massaro D. Tolerance of rats to hyperoxia. Lung antioxidant enzyme gene expression. J Clin Invest 1993; 91: 499-508.

24. Pietarinen-Runtti P, Raivio KO, Saksela M, Asikainen TM, Kinnula VL. Antioxidant enzyme regulation and resistance to oxidants of human bronchial epithelial cells cultured under hyperoxic conditions. Am J Respir Cell Mol Biol 1998; 19: $286-292$

25. Kinnula VL, Crapo JD, Raivio KO. Generation and disposal of reactive oxygen metabolites in the lung. $L a b$ Invest 1995; 73: 3-19.

26. Shull S, Heintz NH, Periasamy M, et al. Differential regulation of antioxidant enzymes in response to oxidants. J Biol Chem 1991; 266: 24398-24403.

27. Asikainen TM, Raivio KO, Saksela M, Kinnula VL. Expression and developmental profile of antioxidant enzymes in human lung and liver. Am J Respir Cell Mol Biol 1998; 19: 942-949.
28. Kinnula VL, Crapo JD. Superoxide dismutases in malignant cells and human tumours. Free Radic Biol Med 2004; 36: 718-744.

29. Comhair SA, Bhathena PR, Dweik RA, Kavuru M, Erzurum SC. Rapid loss of superoxide dismutase activity during antigen-induced asthmatic response. Lancet 2000; 355: 624 .

30. Fattman CL, Schaefer LM, Oury TD. Extracellular superoxide dismutase in biology and medicine. Free Radic Biol Med 2003; 35: 236-256.

31. Gilks CB, Price K, Wright JL, Churg A. Antioxidant gene expression in rat lung after exposure to cigarette smoke. $\mathrm{Am}$ J Pathol 1998; 152: 269-278.

32. Rahman I, van Schadewijk AA, Hiemstra PS, et al. Localization of gamma-glutamylcysteine synthetase messenger RNA expression in lungs of smokers and patients with chronic obstructive pulmonary disease. Free Radic Biol Med 2000; 28: 920-925.

33. Neurohr C, Lenz AG, Ding I, Leuchte H, Kolbe T, Behr J. Glutamate-cysteine ligase modulatory subunit in BAL alveolar macrophages of healthy smokers. Eur Respir $J$ 2003; 22: 82-87. 